\title{
$5-2014$
}

\section{Multi-Muscle FES Force Control of the Human Arm for Arbitrary Goals}

Eric M. Schearer

Cleveland State University, e.schearer@csuohio.edu

Yu-Wei Liao

Northwestern University

Eric J. Perreault

Northwestern University

Matthew C. Tresch

Northwestern University

William D. Memberg

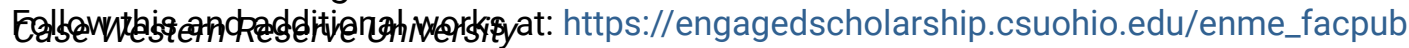

Part of the Biomechanical Engineering Commons

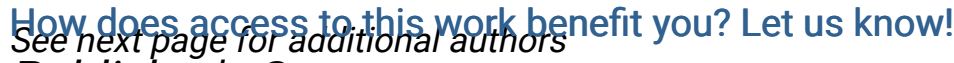

Publisher's Statement

(C) 2014 IEEE. Personal use of this material is permitted. Permission from IEEE must be obtained for all other users, including reprinting/ republishing this material for advertising or promotional purposes, creating new collective works for resale or redistribution to servers or lists, or reuse of any copyrighted components of this work in other works.

\section{Original Citation}

Schearer, E. M., Yu-Wei Liao, Perreault, E. J., 2014, "Multi-Muscle FES Force Control of the Human Arm for Arbitrary Goals," Neural Systems and Rehabilitation Engineering, IEEE Transactions on, 22(3) pp. 654-663.

This Article is brought to you for free and open access by the Mechanical Engineering Department at EngagedScholarship@CSU. It has been accepted for inclusion in Mechanical Engineering Faculty Publications by an authorized administrator of EngagedScholarship@CSU. For more information, please contact library.es@csuohio.edu. 


\section{Authors}

Eric M. Schearer, Yu-Wei Liao, Eric J. Perreault, Matthew C. Tresch, William D. Memberg, Robert F. Kirsch, and Kevin M. Lynch

This article is available at EngagedScholarship@CSU: https://engagedscholarship.csuohio.edu/enme_facpub/303 


\title{
Multi-Muscle FES Force Control of the Human Arm for Arbitrary Goals
}

\author{
Eric M. Schearer, Student Member, IEEE, Yu-Wei Liao, Student Member, IEEE, Eric J. Perreault, \\ Matthew C. Tresch, William D. Memberg, Robert F. Kirsch, Member, IEEE, and Kevin M. Lynch, Fellow, IEEE
}

\begin{abstract}
We present a method for controlling a neuroprosthesis for a paralyzed human arm using functional electrical stimulation (FES) and characterize the errors of the controller. The subject has surgically implanted electrodes for stimulating muscles in her shoulder and arm. Using input/output data, a model mapping muscle stimulations to isometric endpoint forces measured at the subject's hand was identified. We inverted the model of this redundant and coupled multiple-input multiple-output system by minimizing muscle activations and used this inverse for feedforward control. The magnitude of the total root mean square error over a grid in the volume of achievable isometric endpoint force targets was $11 \%$ of the total range of achievable forces. Major sources of error were random error due to trial-to-trial variability and model bias due to nonstationary system properties. Because the muscles working collectively are the actuators of the skeletal system, the quantification of errors in force control guides designs of motion controllers for multi-joint, multi-muscle FES systems that can achieve arbitrary goals.
\end{abstract}

Index Terms-Force control, neural prosthesis, neuromuscular stimulation, system identification.

\section{INTRODUCTION}

F UNCTIONAL electrical stimulation (FES) is a method to restore lost function to persons with paralysis. Although FES has had success in some applications [1]-[3], there remain many challenges. Among these challenges is exploiting the full capability of the musculoskeletal system to perform a

Manuscript received December 06, 2012; revised May 03, 2013; accepted August 09, 2013. Date of publication October 07, 2013; date of current version April 28, 2014. This work was supported by the National Science Foundation (NSF) under Grant 0932263, in part by the National Institutes of Health (NIH) National Institute of Neurological Disorders and Stroke (NINDS) under Grant N01-NS-5-2365, and in part by the NSF Graduate Fellowship DGE-0824162. This work was presented in part at International Conference on Robotic Automation, 2012.

E. M. Schearer and Y. Liao are with the Department of Mechanical Engineering, Northwestern University, Evanston, IL 60208 USA.

E. J. Perreault and M. C. Tresch are with the Department of Biomedical Engineering, Northwestern University, Evanston, IL 60208 USA, and also with the Department of Physical Medicine and Rehabilitation, Northwestern University, Evanston, IL 60611 USA.

W. D. Memberg is with the Department of Biomedical Engineering, Case Western Reserve University, Cleveland, OH 44106 USA.

R. F. Kirsch is with the Department of Biomedical Engineering, Case Western Reserve University, Cleveland, OH 44106 USA, and with the Louis Stokes Veterans Affairs Medical Center, Cleveland, OH 44106 USA, and also with the Department of Orthopedics, MetroHealth Medical Center Cleveland, OH 44109 USA.

K. M. Lynch is with the Department of Mechanical Engineering, Northwestern University, Evanston, IL 60208 USA, and also with the Northwestern Institute on Complex Systems, Northwestern University, Evanston, IL 60208 USA. wide range of tasks. Complex movements such as reaching require the coordination of multiple muscles acting across multiple joints of the skeletal system.

Although controlling multiple muscles with FES potentially provides flexible motor control, that potential has not yet been fully realized. FES applications requiring multiple muscles have generally used fixed muscle activation patterns. For instance, the Freehand System [4] provides users control of their hand but does so by having only a few stereotyped movements. FES controllers for walking [5] and cycling [6] also use stereotyped movements. While controllers for stereotyped movements have restored some function, there is clearly a need for flexible control strategies that can achieve any arbitrary goal subject to the constraints of the musculoskeletal system.

There are many challenges to address when designing such a flexible FES controller with multiple muscles. First, unlike typical serial-chain robotic manipulators, the control of different degrees-of-freedom is not decoupled in human limbs: muscles usually act across multiple degrees-of-freedom. Further, with a large number of muscles needed for flexible control, there are many redundant ways to achieve a given task. Finally, with an increasing number of stimulated muscles, there is a potential increase in the nonlinear interactions between muscles due to current spillover and connective tissue interactions between nearby muscles [7], [8]. The goal of the present study is to design and evaluate a feedforward FES controller for the production of flexible motor outputs that addresses these potential challenges.

Previous studies have designed flexible FES controllers with multiple muscles in order to produce limb movements [9], [10]. They use an optimization of effort or power consumption to specify muscle activations as has been suggested in human motor control literature [11]. While these studies are important in achieving the ultimate aim of restoring flexible motions via FES, they offer only superficial understanding of the many sources of error in multiple-muscle FES control. Limb movements resulting from FES depend on the complex nonlinear dynamics of the musculoskeletal system. Because of this complexity, it is difficult to evaluate the contribution of different sources of error to FES performance when measuring limb movements.

In the present study, we evaluate the performance of a flexible FES controller using multiple muscles to produce isometric forces. This is an important preliminary step in achieving flexible motion control. Since measuring isometric forces avoids contributions of complex limb dynamics to evoked motor outputs, we can readily evaluate the contribution of different sources of error to FES performance. Another study 
TABLE I

STIMULATION ELECTRODES USED

\begin{tabular}{|c|c|c|c|c|c|c|}
\hline & Electrode Placement & Abbreviation & Approximate Function & Type & Current Amplitude (mA) & Max Pulse Width $(\mu \mathrm{s})$ \\
\hline 3 & $\begin{array}{l}\text { Thoracodorsal nerve } \\
\text { Latissimus dorsi }\end{array}$ & Th & Arm adduction & Nerve cuff & 0.8 & 25 \\
\hline 4 & $\begin{array}{l}\text { Long thoracic nerve } \\
\text { Serratus anterior }\end{array}$ & LT & Scapular abduction & Nerve cuff & 1.4 & 12 \\
\hline 5 & $\begin{array}{l}\text { Musculocutaneous nerve } \\
\text { Biceps, brachialis }\end{array}$ & M1 & Elbow flexion & Nerve cuff & 0.8 & 45 \\
\hline 8 & Lower pectoralis & LPec & Shoulder horizontal flexion & Intramuscular & 18.0 & 115 \\
\hline 9 & Upper pectoralis & UPec & Shoulder horizontal flexion & Intramuscular & 20.0 & 70 \\
\hline
\end{tabular}

[12] investigates isometric force control of the thumb, but does not thoroughly investigate the various sources of error of the controller.

In particular, the goals of this study are to quantify the total error in multi-muscle force control, quantify the relative contributions of random error due to trial-to-trial variability and of model bias to the total error, and to quantify the contributions of different sources of model bias in multi-muscle force control. These results provide bounds on the accuracy of the total force applied to the skeletal system by mutliple muscles. These multi-muscle combinations are the actuators that evoke movements of the skeleton. Understanding sources of error at the muscle actuator level guides further development of motion controllers.

\section{METHODS}

In the first four subsections of Section II we describe the experimental subject who participated in this study, the input/ output model that predicts the force at the subject's hand given stimulation inputs to the implanted muscles, a method for identifying this subject-specific model, and the design of an optimization-based feedforward controller based on the identified model. The final three subsections of Section II specifically address the main goals of the study. We describe the experiments used to characterize the total error of the controller over the space of achievable endpoint forces, the experiments to quanitfy the relative contributions of random error due to trial-to-trial variability and of model bias, and the methods used to quantify the possible sources of model bias.

\section{A. Subject}

A 54-year-old female who sustained a hemisection of the spinal cord at the C1-C2 level from a gunshot wound in 1994 participated in this study. She cannot move her right arm, but she has some sensation and pain hypersensitivity. She experiences hypertonia in some of her arm muscles. More details on the subject are included in [13] (Subject 1). Protocols used for research with this subject were approved by the internal review boards at Northwestern University (IRB NO. STU00018382) and MetroHealth Medical Center (IRB NO. 04-00014).

The subject has an implantable stimulator-telemeter [4], [14], [15] located in her abdomen for stimulating muscles in her right arm and shoulder complex. The device has 12 leads that carry current to 12 stimulation electrodes. Three of the leads are attached to intramuscular electrodes [16], which are surgically implanted in muscles. The remaining nine leads are attached to nerve cuff electrodes [17], each of which is wrapped around a nerve that activates one or more muscles. Three leads carry current to electrodes on the cuff around the radial nerve, and two leads carry current to the cuff around the musculocutaneous nerve. We only used one stimulation electrode for each of these nerves leaving three of the 12 stimulation electrodes unused. We refer to each muscle or group of muscles stimulated by a single electrode as a muscle group. In this experiment we used the nine muscle groups shown in Table I. Fig. 1 shows the implanted system and approximate locations of the stimulation electrodes. The subject also has a second implanted device that controls wrist and hand movements, but it was not used in this study.

Power and control signals are sent to the implanted unit through the skin via an inductive radio-frequency link [4], [14], [15]. Stimulation to each muscle group used bi-phasic, charge-balanced pulses delivered at $13 \mathrm{~Hz}$. This frequency was selected to produce reasonably fused contractions while minimizing muscle fatigue. The stimulation amplitude was fixed at a different level for each muscle group (see Table I), while pulse duration could be varied between $0-200 \mu \mathrm{s}$, providing a means to control the force generated by each muscle group. The maximum pulse width for each muscle group was selected 


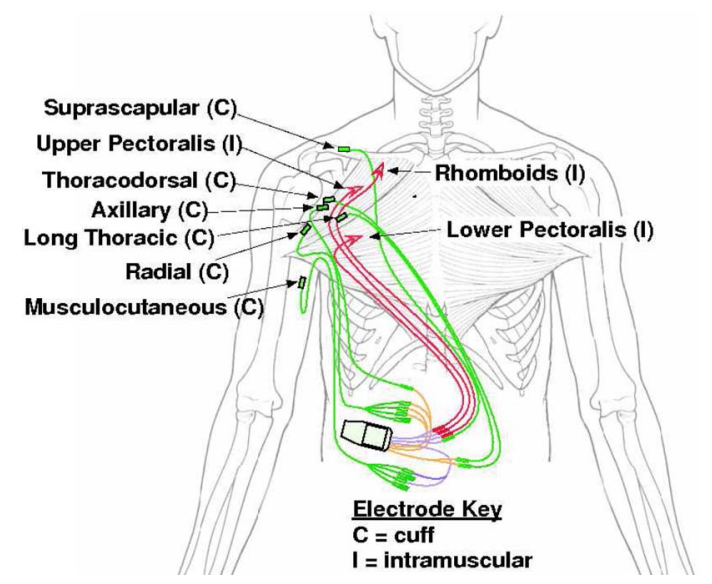

Fig. 1. Implanted stimulator-telemeter device with the 12 stimulation electrodes. A single wire is shown leading to the radial nerve which has three electrodes, and a single wire is shown leading to the musculocutaneous nerve, which has two electrodes, so only nine electrodes are visible in this figure. All other electrodes are single-channel inputs. Nerve-cuff electrodes are marked with green rectangles and green wires and intramuscular electrodes are marked with red arrows and red wires.

to prevent the subject from feeling pain, to prevent spill over to activate other muscles, and when no further force could be achieved with a larger pulse width.

\section{B. Modeling Approach}

Our model predicts the 3-D steady-state force output measured at the hand, which we refer to as the endpoint force, given a set of stimulation inputs to each muscle group. The stimulation input to each muscle group is the pulse width of the initial phase of the bi-phasic charge-balanced pulse. This model was used to estimate the set of all achievable endpoint forces and, through inversion, to determine the stimulation inputs required to achieve a desired endpoint force.

During isometric conditions, there is a linear mapping between muscle force and endpoint force. In contrast, the stimulation-force properties of muscles are nonlinear. Combining these effects, we used a nonlinear mapping (1) to describe the relationship between muscle stimulation inputs $\mathbf{u} \in \mathbb{R}^{9}$ and the endpoint force $\mathbf{f}=\left[f_{x}, f_{y}, f_{z}\right]^{\mathrm{T}}$

$$
\mathbf{f}=A(\mathbf{q}) \mathbf{g}(\mathbf{u}, \mathbf{q})
$$

where the directions of the three components of $f$ are shown by the right-handed frame in Fig. 2. The arm configuration $\mathbf{q} \in$ $\mathbb{R}^{5}$ is the vector of three shoulder joint angles and two elbow angles. The configuration-dependent linear transformation from muscle forces to endpoint forces $A(\mathbf{q}) \in \mathbb{R}^{3 \times 9}$ accounts for the kinematic Jacobian of the arm and the moment arms of the muscle groups about the joints. The columns of $A$ represent the contributions of the individual muscle groups to each of the three components of endpoint force at $100 \%$ stimulation. The nonlinear mapping $\mathbf{g}(\mathbf{u}, \mathbf{q}) \in \mathbb{R}^{9}$ from each of the stimulation inputs to each of the muscle forces is

$$
\mathbf{g}(\mathbf{u}, \mathbf{q})=\left[g_{1}\left(u_{1}, \mathbf{q}\right), g_{2}\left(u_{2}, \mathbf{q}\right), \ldots, g_{9}\left(u_{9}, \mathbf{q}\right)\right]^{\mathrm{T}}
$$

where $g_{j}\left(u_{j}, \mathbf{q}\right)$ is the mapping from the $j$ th stimulation input to the $j$ th muscle force. We refer to this mapping as the muscle

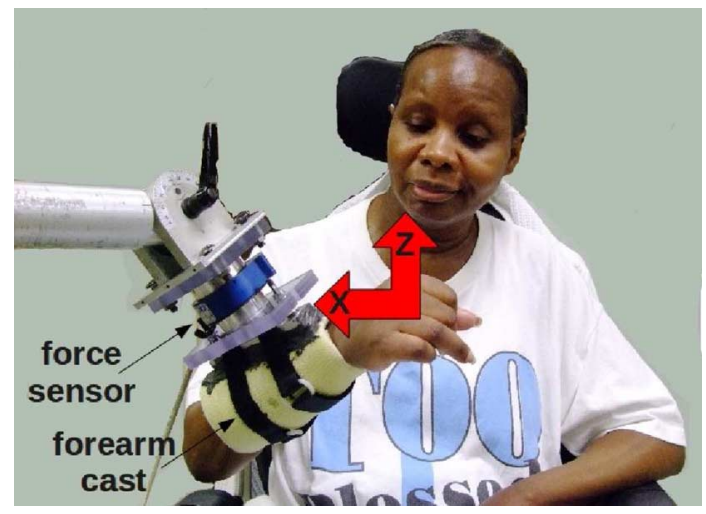

Fig. 2. Experimental setup and coordinate frame.

recruitment curve. In general, $\mathbf{f}, A$, and $\mathbf{g}$ all depend on the configuration of the arm q, but for these isometric experiments conducted at a single configuration we drop the dependence on $q$ and write

$$
\mathbf{f}=A \mathbf{g}(\mathbf{u}) \text {. }
$$

Note that (3) does not include dynamics as we studied only steady-state endpoint forces, ignoring transients. Equation (3) is time-invariant as we have taken care in the experiments to prevent time-varying fatigue from playing a role.

We cannot directly measure the forces that the muscles exert on the skeleton, so we use a proxy in defining $\mathbf{g}(\mathbf{u})$. We define $\tilde{f}_{j} \in \mathbb{R}$ as the magnitude of the endpoint force when the $j$ th input is stimulated. If the mapping from muscle forces to endpoint force is linear, and we only stimulate one muscle group, then the direction of the endpoint force is nearly constant for different stimulation levels, and the magnitude of the endpoint force is proportional to muscle force. In preliminary trials, we verified that the force direction remained approximately constant when increasing the stimulation level. Since we can measure the magnitude of the endpoint force when stimulating the muscle groups individually, we use the endpoint force magnitude in our model instead of the force exerted on the skeleton by the muscle group.

The relationship between each stimulation input and the corresponding magnitude of the endpoint force output $\tilde{f}_{j}$ is nonlinear. We chose a sigmoid function to model this relationship because it is nonlinear and monotonic

$$
\tilde{f}_{j}=\frac{a_{j}}{1+e^{b_{j}\left(c_{j}-u_{j}\right)}}-\frac{a_{j}}{1+e^{b_{j} c_{j}}}
$$

where for the $j$ th muscle group, $a_{j} \in \mathbb{R}$ is the maximum output of the sigmoid function, $b_{j} \in \mathbb{R}$ is proportional to the slope of the sigmoid function at $50 \%$ of the maximum output, and $c_{j} \in \mathbb{R}$ is the input at which the sigmoid function outputs $50 \%$ of its maximum output. The second term on the right-hand side forces the output to be zero when the input is zero. We then normalize $\tilde{f}_{j}$ by the endpoint force magnitude when the maximum stimulation pulse width is applied. Equation (3) uses this normalized value for $g_{j}\left(u_{j}\right)$. We normalize so that each $g_{j}\left(u_{j}\right) \in[0,1]$ and represents muscle activation.

Next we explain how to identify $a_{j}, b_{j}$, and $c_{j}$ for each muscle group and $A$ for the arm configuration tested. 


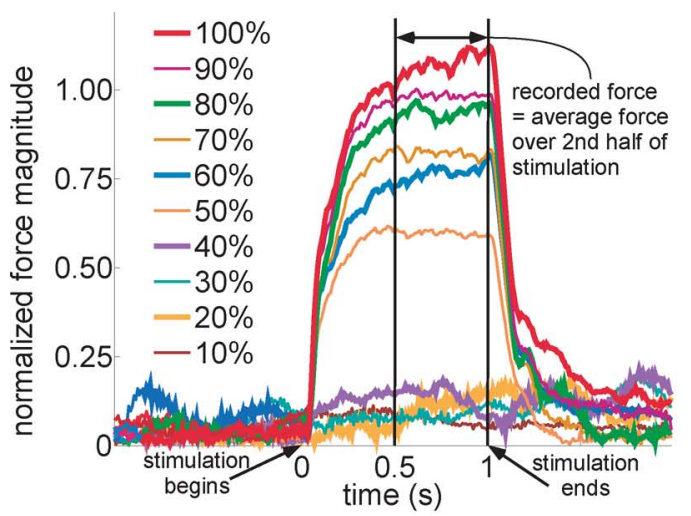

(a)

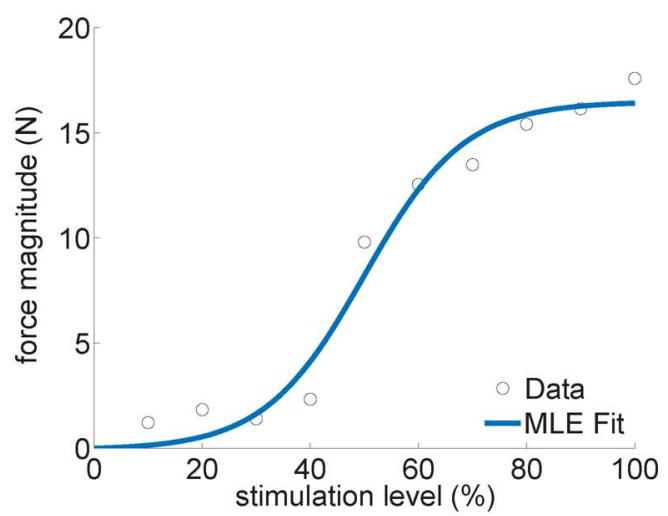

(b)

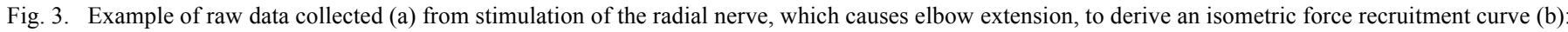

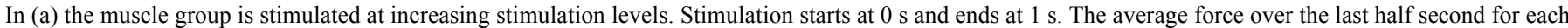

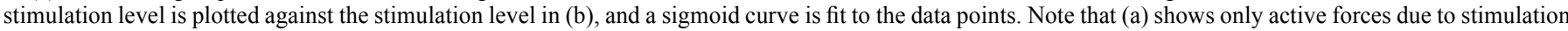
of the muscle group. It does not include passive effects such as gravity or muscle stiffness.

\section{Model Identification Experiments}

The model identification experiments took place on Day 1 of two days of testing. The subject's right forearm was strapped into a cast rigidly attached to a force sensor (JR3 Model 67M25A3-I40) as shown in Fig. 2. The subject's arm was at approximately $45^{\circ}$ of shoulder elevation measured in a vertical plane rotated by $70^{\circ}$ from the coronal plane, $55^{\circ}$ of shoulder internal rotation, $90^{\circ}$ of elbow flexion, and zero forearm pronation/supination. We chose this configuration $[q$ in (1)] because it is useful in everyday tasks and a configuration for which we expected the arm to exert significant endpoint forces in each of the measurement directions.

To identify the model described by (3) and (4) we used a method similar to the steady-state step response method [18]. We stimulated each muscle group at discrete stimulation pulse widths and recorded the resulting steady-state endpoint force. We used a fixed transformation from the sensor coordinate frame to the coordinate frame at the third knuckle of the hand shown in Fig. 2.

Each of the nine muscle groups was stimulated at constant pulse widths of $10 \%, 20 \%, 30 \%, 40 \%, 50 \%, 60 \%, 70 \%, 80 \%$, $90 \%$, and $100 \%$ of its maximum pulse width for a total of 90 stimulation trials. A 5-s rest period followed each 1-s stimulation. The order of muscle groups stimulated and pulse width levels was randomized. The experiment was separated into ten blocks of nine different muscle groups per block, so each muscle group was only stimulated once during each 54-s block. This allowed each muscle group sufficient time to rest before stimulation in the next block.

An example of evoked force versus time for the ten trials for a muscle group is shown in Fig. 3(a). Each point on the recruitment curve [Fig. 3(b)] is the difference between the force magnitude averaged over the last half second of stimulation and the force magnitude averaged over the second before stimulation began. Subtracting the force measured before stimulation begins makes this a model for the active steady-state force generated by stimulating a muscle group and eliminates passive effects such as gravity or muscle stiffness. Generally the force magnitudes in Fig. 3(a) plateau after $0.5 \mathrm{~s}$ of stimulation. Fluctuations in the force magnitude for $60 \%, 80 \%$, and $100 \%$ stimulation are due to movement that results from breathing. The model treats these fluctuations as noise. Maximum likelihood was used to estimate the parameters $a_{j}, b_{j}$, and $c_{j}$ for each recruitment curve.

Because we stimulated the muscle groups one-by-one, we solved for each column of $A$ in (3) separately. This was done by a linear least squares fit with no intercept term

$$
A_{j}=F_{j} \tilde{\mathbf{g}}_{\mathbf{j}}^{\dagger}
$$

where $A_{j} \in \mathbb{R}^{3 \times 1}$ is the column of $A$ corresponding to the $j$ th muscle group (Fig. 4), $F_{j} \in \mathbb{R}^{3 \times 10}$ is the matrix of endpoint force vectors (ten vectors, one for each stimulation level) corresponding to the $j$ th muscle group, $\tilde{g}_{\mathbf{j}} \in \mathbb{R}^{1 \times 10}$ is a vector of normalized force magnitudes (one for each stimulation level) corresponding to the $j$ th muscle group, and $\tilde{\mathbf{g}}_{\mathbf{j}}^{\dagger}$ is the Moore-Penrose pseudo-inverse of $\tilde{\mathbf{g}}_{\mathbf{j}}$.

\section{Feedforward Controller}

Using the identified model, we developed a feedforward controller that determines the stimulation inputs that will produce a desired active endpoint force.

Given a target force $\mathbf{f}^{\mathbf{t}}$, the controller inverts the model identified above to compute the stimulation inputs $\mathbf{u}$ to be applied to the arm. Because the system is redundant, there is not a unique solution of (3) for $\mathbf{g}(\mathbf{u})$. To resolve the redundancy we minimize the sum of squares of muscle activations subject to the constraints that the model-predicted force equals the target force and that the activations are between zero and one

$$
\begin{aligned}
\text { minimize : } & \|\mathbf{g}(\mathbf{u})\|_{2}^{2} \\
\text { subject to : } & A \mathbf{g}(\mathbf{u})=\mathbf{f}^{\mathbf{t}} \\
& g_{j}\left(u_{j}\right) \in[0,1] \quad \forall j .
\end{aligned}
$$

Having obtained the activations $\mathbf{g}(\mathbf{u})$ required to achieve the target endpoint force, the controller inverts the recruitment curve (4) to find each required stimulation input $u_{j}$. There is evidence that the healthy nervous system uses this minimum activation strategy [11]. 


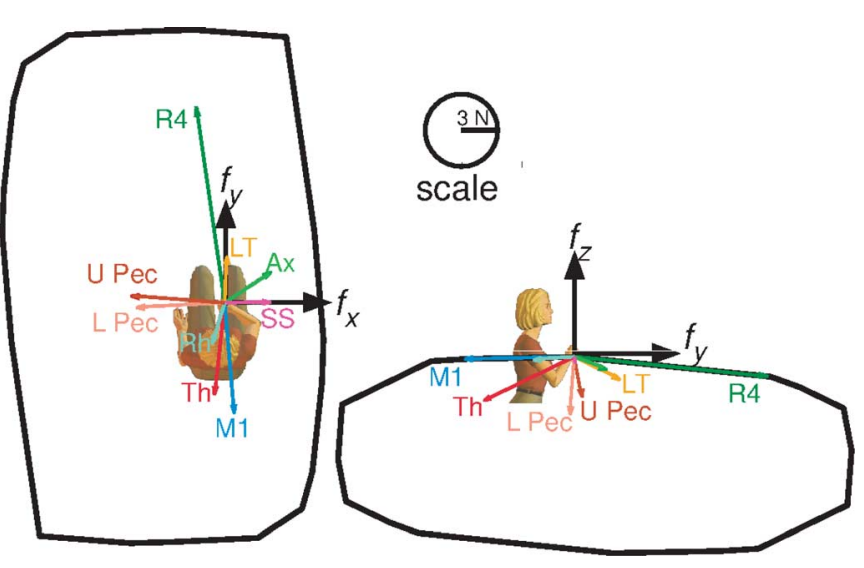

Fig. 4. Graphical description of $A$ in (3): each vector originating from the subject's hand represents the magnitude and direction of the force at the subject's hand when stimulating the corresponding muscle group at $100 \%$. Each vector is a column of $A$. Only six muscle groups are labelled in the view on the right because of space considerations. SS is not visible in the view on the right because it has very small $f_{y}$ and $f_{z}$ components. The black rings bound the set of achievable endpoint forces in each plane.

\section{E. Total Error in Multi-Muscle Force Control}

We evaluated the accuracy of our controller using a grid of evenly-spaced endpoint force targets over the 3-D range of achievable forces. To determine the range of achievable endpoint forces we assumed that each muscle group acts independently, and can produce a set of forces defined by the line segment between zero and the maximum 3-D force measured during the characterization of its recruitment curve. The set of all achievable forces is then the Minkowski sum of the nine sets of achievable forces of the individual muscle groups. The "volume" of achievable forces is a convex region and can be seen in 2-D slices (Fig. 5).

We constructed a grid of targets (Fig. 5) in the $\left[f_{x}, f_{y}, f_{z}\right]$ space with $4.5 \mathrm{~N}$ spacing to fill the volume of achievable endpoint forces. Controller performance trials were conducted on two separate days with $48 \mathrm{~h}$ of rest between sessions. This resulted in 69 targets on Day 1 and 66 new targets on Day 2 for a total of 135 unique endpoint force targets. The orientation of $\left[f_{x}, f_{y}, f_{z}\right]$ coordinate systems in which the targets were computed were different from one day to the next. On Day 1 the order of targets was randomized and divided into three blocks. The Day 2 targets were randomized in two equal blocks. The blocks were run in succession with a short period for data logging between blocks. One trial was run for each target.

Each trial consisted of stimulating the muscle groups corresponding to the desired target for $1 \mathrm{~s}$ followed by $30 \mathrm{~s}$ of rest to limit any effects of fatigue. The measured steady-state force, which was the mean force output over the final $0.5 \mathrm{~s}$ of stimulation, was recorded. We computed the error, which is the difference between the predicted and measured steady-state force output, for each target and computed the root mean square (rms) error over all targets.

\section{F. Random Error and Bias in Multi-Muscle Force Control}

To estimate the relative contributions of random error and bias to the total error, we ran several repeated trials at five different endpoint force targets spanning much of the achievable

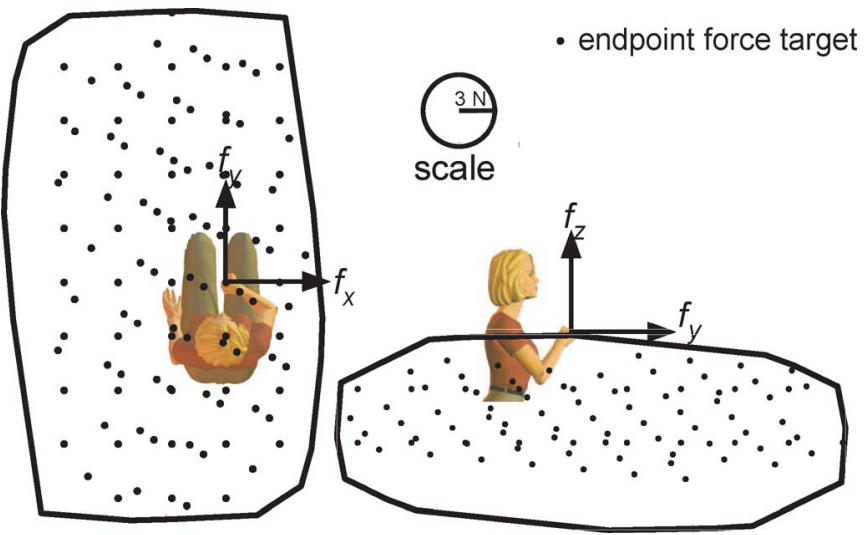

Fig. 5. Target forces in controller performance experiments: Black circles represent targets in the $x y$ and $y z$ planes in endpoint force space. If a vector were drawn from the subject's hand to each black circle, that vector represents the direction and magnitude of the target force. The black rings bound the set of achievable endpoint forces in each plane.

force space (Fig. 8). On Day 1 a single target (Target 1) was repeated 10 times. On Day 2 four additional targets (Targets 2-5) were repeated 15 times each. On Day 2 the trials for the first two targets were run in random order in one block and the trials for the last two targets were then run in random order in a second block. The timing of stimulation and rest were the same as in the previous subsection.

Random error was quantified for each target by computing the covariance of the measured 3-D force for the repeated trials and reporting the square root of the largest eigenvalue. This represents the standard deviation of the force in the direction of largest variance. Bias error was computed as the difference between the target and the mean of the measured force over the repeated trials at each target.

\section{G. Sources of Model Bias in Multi-Muscle Force Control}

Our results indicated that bias errors were larger than random errors for our controller. We therefore investigated three possible sources of the observed bias. The first potential source is due to system nonstationarity present between the system identification experiments on Day 1 and the subsequent controller performance experiments on Days 1 and 2. Possible sources of nonstationarity include fatigue or experimental outliers associated with nonstationary events such as muscle spasms or postural changes during the system identification process. The second possible source of bias is nonlinear interactions between muscle groups, which are not considered in our model (3). These may arise from current spillover or force transmission between muscle groups. The third source of bias is poor model fits due to an insufficient amount of data.

To evaluate the effects of nonstationarities we refit our linear model mapping muscle activations to endpoint forces to the controller performance experiment data. Then we compared the refit model's ability to predict endpoint forces to that of the model that was fit to the original system identification data. The parameters of the model $[A$ in (3)] were identified using data from 100 trials randomly-selected from the data set of 135 trials, and the model's performance was evaluated on 30 other randomly-selected trials. The superior performance of the refit 


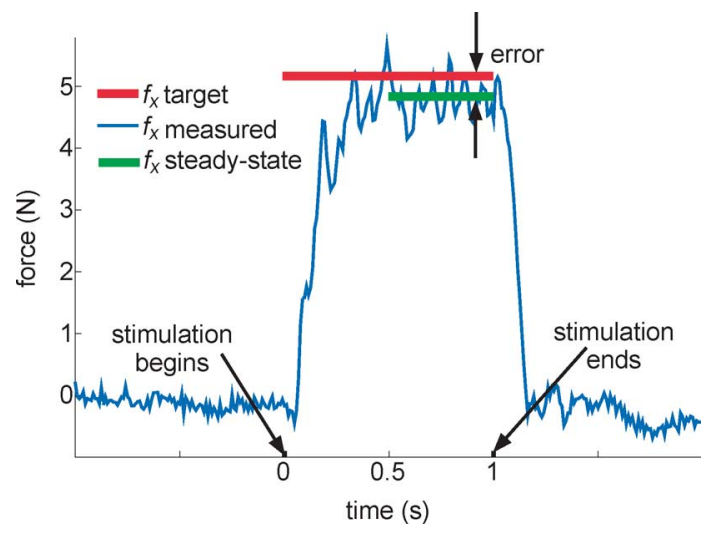

Fig. 6. Measured force in the $x$ direction versus time for a typical controller performance experiment: The steady-state force is the average of the measured force during the final half second of stimulation.

model, if any, indicates differences between the original system identification data set and the controller performance data. For instance, if muscle properties changed between the system identification experiments and the controller performance experiments, the new refit model should perform better. We attribute these differences to nonstationary system properties.

The process for refitting the model to the controller performance experiment data was as follows. As the controller performance experiment data includes no information on muscle groups stimulated individually, we could not directly recompute recruitment curves. To indirectly recompute recruitment curves we fit a Gaussian process model (GPM) [19], which is a nonlinear function approximator, to the entire 135 data point set. Using the GPM we created simulated single muscle group data and computed recruitment curves based on the simulated data. Given stimulation inputs from the controller performance experiment data, we computed the corresponding muscle activations using the recomputed recruitment curves. We then refit our linear model (3) to 100 randomly-selected trials and evaluated its predictions for 30 other randomly-selected trials.

This cross-validation process was repeated 1000 times, yielding 1000 new linear models. To quantify the effects of nonstationarities we compared the mean rms error over these models to the error in the controller performance experiment.

To evaluate the effects of nonlinear interactions, we fit Gaussian process models to the same data sets that were used to refit the linear models, yielding 1000 GPMs. The GPMs predict the endpoint force given the muscle activation. If the GPM, which can capture a wide range of nonlinear interactions between muscles, predicts endpoint forces better than the linear model, it would suggest that nonlinear interactions between muscles significantly affected controller performance. We used the same cross-validation process for the GPMs as was described for the linear models and compared the average rms error of the GPM models to the average rms error of the linear models to evaluate the effects of nonlinear interactions.

Finally, the influence of the amount of data available for fitting the model parameters was assessed by repeating the fitting process with randomly selected data sets having $20,30,40,50$, $60,70,80,90$, and 100 data points. This process was performed for both the GPM model and the reestimated linear model, and

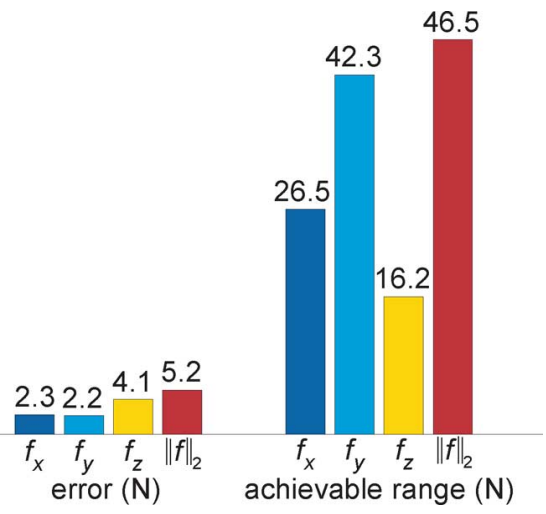

Fig. 7. RMS errors in endpoint force compared to the range of achievable endpoint forces over both days of controller performance experiments.

performance of each model was cross-validated using 30 randomly selected trials not used to fit the model. This process was repeated 1000 times for each size data set.

We trained Gaussian process models using the GPML toolbox [20] for MATLAB. Our GPMs used a zero mean function, a squared exponential covariance function, and a Gaussian likelihood function. The model's hyperparameters - the input length scale, the output covariance and the noise level-were determined by maximizing the marginal likelihood of the data, which balances fitting the data well with avoiding model complexity.

\section{RESULTS}

\section{A. Total Error in Multi-Muscle Force Control}

The results of a typical controller performance trial are shown in Fig. 6. Shortly after stimulation began the measured force began to rise and eventually oscillated around a steady-state value. When stimulation ended, the measured force returned close to its pre-stimulation level. Similar trials were conducted with targets over the entire space of achievable endpoint forces.

The magnitude of rms error of the controller over the 135 evenly-spaced targets was $5.2 \mathrm{~N}$ or $11 \%$ of the range of achievable endpoint forces (Fig. 7). The largest rms error was in the vertical $(z)$ direction. The error was $4.1 \mathrm{~N}$ or $26 \%$ of the range of achievable vertical forces. The results were similar over both days of testing as the magnitude of rms error on Day 1 was 5.3 $\mathrm{N}$ and on Day 2 was $5.1 \mathrm{~N}$.

The largest errors occurred when undershooting targets in the vertical $(z)$ direction. This means that muscle groups producing force in the vertical direction did not produce as much force as the identified model predicted. The largest errors coincided with stimulation of lower pectoralis and the thoracodorsal nerve which were the primary producers of vertical force at this arm pose (see Fig. 4). The reasons for these errors are discussed further in Section III-C.

\section{B. Random Error and Bias in Multi-Muscle Force Control}

The total error reported above reflects both errors due to bias in the feedforward controller and random errors due to variability of force production across trials. We evaluated the contributions of these different sources of error by running repeated trials for five different endpoint force targets. 


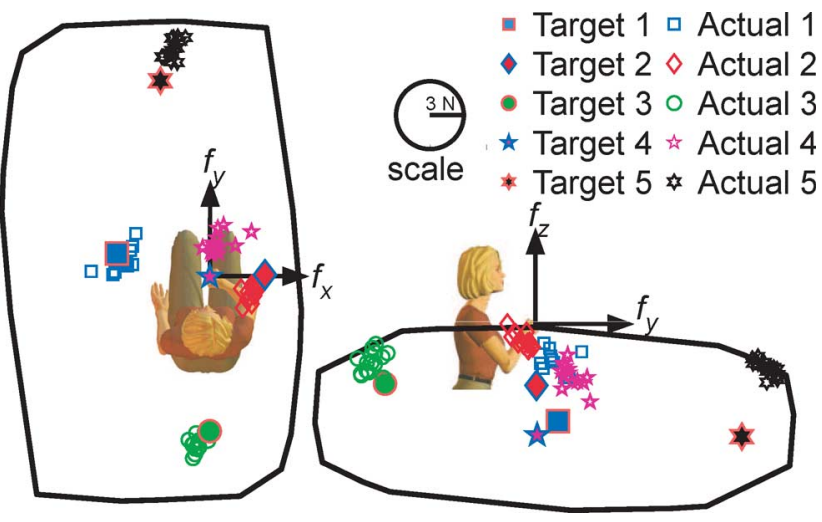

Fig. 8. Scatter plots of repeated controller performance experiments in the $x y$ plane (left) and the $y z$ plane (lower right): Large closed symbols represent the endpoint force targets, and smaller open symbols represent the actual endpoint force measured in repeated experiments. If a vector were drawn from the subject's hand to each symbol, that vector represents the direction and magnitude of the target force for a large closed symbol or the measured force for a smaller open symbol. The black rings bound the set of achievable endpoint forces in each plane. Target 1 was tested on Day 1, and the other four targets were tested on Day 2.

Errors due to model bias were larger than errors due to trial-to-trial variability. The forces observed on repeated control trials attempting to produce five different force targets are shown in Fig. 8. For each target, each of the repeated trials used the identical muscle stimulation pattern. As can be seen in the figure, the desired force and the distribution of forces actually produced for each target are different. These errors due to bias ranged between 2.3-6.0 N (rms), corresponding to $5 \%-13 \%$ of the total range of achievable endpoint forces. The random error due to trial-to-trial variability, as measured by the distribution of measured forces for each target, was relatively smaller, ranging between 0.9 to $1.6 \mathrm{~N}$, or $2 \%-3 \%$ of the total range of achievable endpoint forces.

\section{Sources of Model Bias in Multi-Muscle Force Control}

Because model bias errors were larger than random errors we further investigated the sources of model bias. We quantified model bias due to nonstationary system properties, nonlinearity of muscle interactions, and the use of limited data in system identification.

To estimate model bias due to nonstationary system properties, we reestimated our model 1000 times using different randomly-selected trials from the controller performance experiments. We compared the average rms error of the refit models to the rms error of the original controller.

Reestimating the model reduced the magnitude of the total rms error to $2.7 \mathrm{~N}$ or $2.5 \mathrm{~N}$ less than the error of the original model. We attribute this $2.5 \mathrm{~N}$ difference to nonstationary system properties. The error in the vertical force component was reduced to $1.1 \mathrm{~N}$, or $3.0 \mathrm{~N}$ less than the vertical component of the error of the original model. This demonstrates that the systematic undershoot mentioned in Section III-A was greatly reduced using the reestimated model. Smaller error reductions were observed in the two horizontal directions. Almost all parameters in the refit linear models were significantly different than those originally identified for every muscle group.

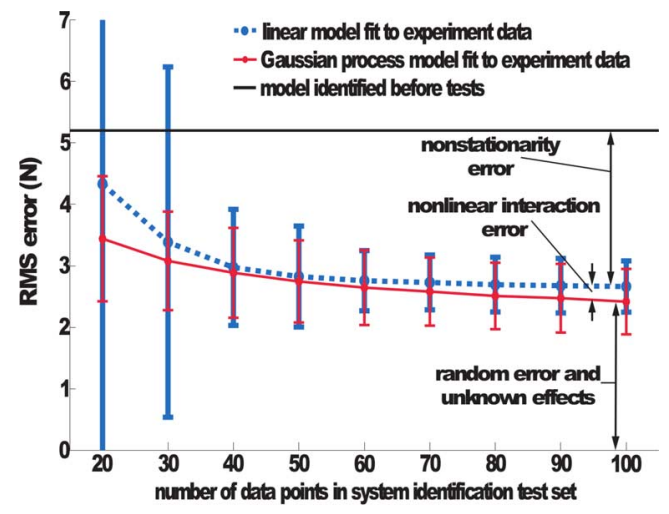

Fig. 9. Sources of error in multi-muscle endpoint force control. 1000 linear models and 1000 GPMs were fit to randomly-selected test sets from the controller performance data set for increasing numbers of data points in the test set. The average rms error of the models in predicting endpoint forces for data not in the test set is represented by blue circles for the linear models and red circles for the GPMs. The error bars represent two standard deviations away from the average of the 1000 models. The solid horizontal black line represents the rms error of the controller that used the model identified on 90 data points prior to the controller performance experiments.

Despite nonstationarity in all muscle groups, especially large errors coincided with stimulation of lower pectoralis as mentioned in Section III-A. The decrease in error with the refit models was primarily in the vertical direction to which the lower pectoralis contributed significantly. The reason for this is that the nonstationarity in the lower pectoralis recruitment curve occurred at a stimulation level that was very frequently used. The range of stimulation of other muscle groups was more uniform during the controller performance experiments, so the effects of nonstationarity were diminished.

We found that there was only a modest reduction in error when the second source of model bias-nonlinear interactions between muscle groups - was considered. To show this we fit a Gaussian Process Model (GPM) to each of 1000 data sets randomly selected from the controller performance experiment data. A GPM can account for nonlinearities as detailed in Section II-G. The average rms error of these 1000 GPMs was compared to the average rms error of the 1000 linear models described above. The reduction in average rms error was from $2.7 \mathrm{~N}$ using the linear model to $2.4 \mathrm{~N}$ using the nonlinear GPM (Fig. 9). The difference between the average rms error of the GPMs and the average rms error of the linear models was not statistically significant (see error bars in Fig. 9). This small improvement when using the nonlinear GPM suggests that the forces produced by individual muscle groups combined approximately linearly during the controller performance experiments.

Finally, we examined the consequences of estimating models using limited amounts of data. As shown in Fig. 9, the prediction error steadily decreased with increasing numbers of data trials used to identify the model, up until approximately 40 data points were used. There were minimal improvements in model predictions as data sets were increased beyond this number for models allowing either linear or nonlinear interactions between muscles. 


\section{DISCUSSION}

The goal of this research was to quantify the errors in force productioin associated with using FES to control multiple muscles in the human arm. To eliminate complications associated with modeling the relationship between muscle forces and the resulting motions of a multiple-degree-of-freedom limb, we considered only isometric force generation and an empirically determined linear mapping between the forces generated by each muscle and those measured at the endpoint of the arm. This approach allowed us to quantify the total error associated with multiple muscle control and to estimate the contributions that could be attributed to nonlinear interactions between muscles, nonstationary system properties, model biases resulting from the use of limited data during the estimation process, and random errors.

Quantifying errors in isometric force control is a necessary step toward motion control of multiple joints. The multi-muscle system applies forces that produce torques across multiple joints. The endpoint force we measured is linearly related to torques about the joints of the arm. In quantifying the error in endpoint force relative to the total range of force output, we quantify the expected errors in joint torques relative to the range of possible joint torques. We have quantified the steady-state accuracy of the torque actuators of our system. This is especially useful in using well-known robot arm control strategies which typically command torques to produce desired motions. For instance, in designing a controller where torques are commanded, the expected uncertainty in the torque command can be propogated through a simulation model to predict errors in the motion of the arm.

Our work in isometric force control does not address some factors that may contribute to errors in motion control. We intentionally did not consider the nonlinear dynamics of the skeletal system in order to isolate the effects of muscle force production. Our steady-state analysis also did not consider the dynamics of the muscles themselves. Identifying subject-specific models of the muscle dynamics and skeletal dynamics are critical future steps that will build on our current investigation of isometric force generation by mutliple muscles.

The remainder of the discussion focuses on the specific findings of this study. We found that nonlinear interactions between muscles made only modest contributions to the total error of the controller, while random error and errors due to nonstationary system properties had more substantial contributions. We discuss each of these results and their potential implications to the design of FES controllers below.

\section{A. Nonlinear Interactions Between Muscles}

The finding of minimal contribution of nonlinear interactions between muscles was somewhat surprising as there is evidence to suggest nonlinear interactions exist [7], [8]. This result suggests that nonlinearities due to current spillover to adjacent muscles or due to connective tissue interactions between muscles have minimal effect on force production by multiple muscles. A minimal effect of nonlinear interactions between muscles was recently described in similar studies performed in the rat [21].

The finding that muscle forces add linearly in a human FES system greatly simplifies the design of FES controllers. If nonlinear interactions were present, more complicated models of force production would be required, significantly increasing the complexity of FES controller design. Note that we selected the range of stimulation pulse widths to explicitly avoid current spillover. Further, nonlinear connective tissue interactions between muscles might become more significant at extreme limb configurations, or during more dynamic conditions not considered in these experiments.

\section{B. Errors due to Nonstationary System Properties}

A large source of error in our experiments was due to differences between the forces produced by muscle groups during the initial system identification experiments and during the controller performance experiments. When these differences were accounted for, the total error was reduced by almost $50 \%$. These errors imply that the system was nonstationary. Contributions to this behavior could arise from outliers during the initial system identification data collection process, such as those arising from muscle spasms in patients with SCI, changes in stimulation history, fatigue, or significant random errors between trials. It is difficult to evaluate if such nonstationarities would be expected consistently for FES controllers, because of the limited data available for these experiments. Nonetheless, these results do emphasize the importance of tracking changes in the muscle response to stimulation over time to improve FES controller performance. Such changes might be corrected directly using adaptive control strategies or accounted for more indirectly using robust feedback controllers.

\section{Random Error due to Trial-to-Trial Variability}

Trial to trial variability contributed approximately $1.6 / 5.2 \mathrm{~N}$ $(30 \%)$ of the total error. If the errors due to any nonlinear interactions and changes in estimated muscle properties are accounted for, trial-to-trial variability contributed 1.6/2.5 N (64\%) of the total error. This random error might in part be due to nonstationarities in the physiological state of the musculoskeletal system during the period of the controller performance trials. This could arise from fatigue, changes in arousal, reflexes, or muscle spasms.

In addition to these physiological sources, it is also possible that some of this random error was due to measurement error. As seen in the raw data trial of Fig. 6, there were considerable fluctuations in the forces that were measured, due in part to the patient's respiration. Because of the likely contributions of such measurement errors, it is likely that the random error observed here was an overestimate of the true variability of force production during FES and should be considered as an upper bound on the amount of random error.

\section{Conclusions}

The results of this study describe the performance of a feedforward FES controller for the production of flexible motor outputs in humans. We demonstrated the contributions of different sources of error to the performance of this controller, showing a minimal effect from nonlinear interactions between muscles but greater effects from errors due to nonstationary system properties and from trial-to-trial variability.

Although we were able to account for a substantial portion of the observed errors in FES performance, it is important to 
note that some unexplained, residual error was observed. If we consider that $0.9-1.6 \mathrm{~N}$ of the total error of $2.4 \mathrm{~N}$ for the nonlinear GPM model was due to trial-to-trial variability, then there remains approximately $0.8-1.5 \mathrm{~N}$ of error that we have not accounted for. This residual error might reflect physiological processes, such as muscle fatigue during the controller performance experiments, which were not evaluated systematically in these experiments. It therefore remains possible that the error observed here for feedforward FES control could be reduced further if this residual error could be accounted for and incorporated into the controller.

It is clear that any practical FES system will need to include some form of feedback control to compensate for errors in force production, but it is unclear at what level this feedback should occur. Feedback can be provided at the highest level by the user, who is able to grade the level of stimulation through the user interface [22], [23]. Alternatively, automatic feedback control may be used to correct errors in the output [9], [10], which could be the position of the hand or the joint angles of the arms. Automatic feedback could also be used at the muscle actuator level to correct errors in force and torque [24].

The results of the present study provide important information on the characteristics of the internal errors that need to be considered when designing a feedback controller for a high-degree-of-freedom system such as the human arm.

\section{REFERENCES}

[1] K. T. Ragnarsson, "Functional electrical stimulation after spinal cord injury: Current use, therapeutic effects and future directions," Spinal Cord, vol. 46, pp. 255-274, 2008.

[2] P. H. Peckham and J. S. Knutson, "Functional electrical stimulation for neuromuscular applications," Annu. Rev. Biomed. Eng., vol. 7, pp. 327-360, 2005.

[3] L. R. Sheffler and J. Chae, "Neuromuscular electrical stimulation in neurorehabilitation," Muscle Nerve, vol. 35, no. 5, pp. 562-590, 2007.

[4] B. Smith, P. H. Peckham, M. W. Keith, and D. D. Roscoe, "An externally powered, multichannel, implantable stimulator for versatile control of paralyzed muscle," IEEE Trans. Biomed. Eng., vol. 34, no. 7, pp. 499-508, Jul. 1987.

[5] D. Zhang and K. Zhu, "Modeling biological motor control for human locomotion with functional electrical stimulation," Biol. Cybern., vol. 96, pp. 70-97, 2007.

[6] P. Li, Z. Hou, F. Zhang, M. Tan, H. Wang, Y. Hong, and J. Zhang, "An FES cycling control system based on CPG," in Proc. 31st Annu. Int. Conf. IEEE EMBS, 2009, pp. 1569-1572.

[7] H. Maas and T. G. Sandercock, "Force transmission between synergistic skeletal muscles through connective tissue linkages," J. Biomed. Biotechnol., 2010, 575672.

[8] P. A. Huijing, "Epimuscular myofascial force transmission: A historical review and implications for new research. International Society of Biomechanics Muybridge Award Lecture, Taipei, 2007," J. Biomechan., vol. 42, no. 1, pp. 9-21, 2007.

[9] D. Blana, R. F. Kirsch, and E. K. Chadwick, "Combined feedforward and feedback control of a redundant, nonlinear, dynamic musculoskeletal system," Med. Biol. Eng. Comput., vol. 47, pp. 533-542, 2009.

[10] T. Watanabe, K. Iibuchi, K. Kurosawa, and N. Hoshimiya, "A method of multichannel PID control of two-degree-of-freedom wrist joint movements by functional electrical stimulation," Syst. Comput. Jpn., vol. 34 , no. 5 , pp. $25-36,2003$.

[11] A. H. Fagg, A. Shah, and A. G. Barto, "A computational model of muscle recruitment for wrist movements," J. Neurophysiol., vol. 88, pp. 3348-3358, 2002.

[12] J. L. Lujan and P. E. Crago, "Automated optimal coordination of multiple-DOF neuromuscular actions in feedforward neuroprostheses," IEEE Trans. Biomed. Eng., vol. 56, no. 1, pp. 179-187, Jan. 2009.
[13] K. H. Polasek, H. A. Hoyen, M. W. Keith, R. F. Kirsch, and D. J. Tyler, "Stimulation stability and selectivity of chronically implanted multicontact nerve cuff electrods in the human upper extremity," IEEE Trans. Neural Syst. Rehabil. Eng., vol. 17, no. 5, pp. 428-437, Oct. 2009.

[14] B. Smith, Z. Tang, M. W. Johnson, S. Pourmehdi, M. M. Gazdik, J. R. Buckett, and P. H. Peckham, "An externally powered, multichannel, implantable stimulator-telemeter for control of paralyzed muscle," IEEE Trans. Biomed. Eng., vol. 45, no. 4, pp. 463-475, Apr. 1998.

[15] R. L. Hart, N. Bhadra, F. W. Montague, K. L. Kilgore, and P. H. Peckham, "Design and testing of an advanced implantable neuroprosthesis with myoelectric control," IEEE Trans. Neural Syst. Rehabil. Eng., vol. 19, no. 1, pp. 45-53, Jan. 2011.

[16] W. D. Memberg, P. H. Peckham, and M. W. Keith, "A surgically-implanted intramuscular electrode for and implantable neuromuscular stimulation system," IEEE Trans. Rehabil. Eng., vol. 2, no. 2, pp. 80-91, Jun. 1994.

[17] G. G. Naples and J. T. Mortimer, "A spiral nerve cuff electrode for peripheral nerve stimulation," IEEE Transactions on Biomedical Engineering, vol. 35, no. 11, pp. 905-916, 1988.

[18] W. K. Durfee and K. E. MacLean, "Methods for estimating isometric recruitment curves of electrically stimulated muscle," IEEE Trans. Biomed. Eng., vol. 36, no. 7, pp. 654-666, Jul. 1989.

[19] C. E. Rasmussen and C. K. I. Williams, Gaussian Processes for Machine Learning. Cambridge, MA: The MIT Press, 2006.

[20] C. E. Rasmussen and H. Nickisch, "GPML Gaussian processes for machine learning toolbox," 2010 [Online]. Available: http://mloss.org/ software/view/263/

[21] A. M. Jarc, M. Berniker, and M. C. Tresch, "FES control of isometric forces in the rat hindlimb using many muscles," IEEE Trans. Biomed. Eng., vol. 60, no. 5, pp. 1422-1430, May 2013.

[22] C. Ethier, E. R. Oby, M. J. Bauman, and L. E. Miller, "Restoration of grasp following paralysis through brain-controlled stimulation of muscles," Nature, vol. 485, pp. 368-371, 2012.

[23] J. G. Hincapie and R. F. Kirsch, "Feasibility of EMG-based neural network controller for an upper extremity neuroprosthesis," IEEE Trans. Neural Syst. Rehabi. Eng., vol. 17, no. 1, pp. 80-90, Feb. 2009.

[24] M. A. Frankel, B. R. Dowden, V. J. Mathews, R. A. Normann, G. A. Clark, and S. G. Meek, "Multi-input single-output closed-loop isometric force control using asynchronous intrafascicular multi-electrode stimulation," IEEE Trans. Neural Syst. Rehabil. Eng., vol. 19, no. 3, pp. 325-332, Jun. 2011. 